\title{
NUCLEAR SPIN RELAXATION IN PERIODICALLY PERTURBED SYSTEMS*
}

\author{
J.S. Blicharski AND A. WOLAK \\ Institute of Physics, Jagellonian University, Reymonta 4, 30-059 Kraków, Poland.
}

(Received June 21, 1990)

The effective relaxation time $T_{2 e}$ is calculated in the weak collision case for a system of identical nuclear spins perturbed by periodic sequences of r.f. pulses and dipole-dipole and quadrupole interactions.

PACS numbers: 76.60.-k

\section{Introduction}

The aim of this paper is to calculate effective relaxation time $T_{2 \mathrm{e}}$ for identical nuclear spins in the presence of r.f. multipulse sequences and molecular motions. The effective relaxation times have been considered in a series of papers for periodic sequences of $90^{\circ}$ r.f. pulses [1-12] and r.f. pulses with arbitrary nutation angle [13, 14]. In most of the previous papers only secular part of dipole-dipole interaction has been taken into account. In the present paper we consider the relaxation due to fluctuating secular and nonsecular parts of the dipolar and quadrupolar interactions and multipulse sequences with arbitrary phase and nutation angles.

\section{General theory}

Nuclear spin relaxation and spin dynamics can be described on the basis of the density operator $\boldsymbol{\sigma}$ which obeys Liouville-von Neumann equation of motion [15]:

$$
\frac{\mathrm{d} \sigma}{\mathrm{d} t}=-\mathrm{i}[\mathcal{H}, \boldsymbol{\sigma}]
$$

where $\mathcal{H}$ is spin Hamiltonian, in angular frequency units. The $\mathcal{H}$ is a sum of strong Zeeman interaction of spins with external magnetic field $\boldsymbol{B}_{0}$, interaction

*This work was supported by the CPBP 01.09. theme III.3.2.01 
with radio-frequenccy (r.f.) field $B_{1}$, static part of spin interactions and time dependent perturbation $\mathcal{H}(t)$, due to random fluctuating part of spin interactions.

Using transformation into interaction frame (rotating toggling frame-RTF) [6] and two following iteraction in (1), as well as Fano expansion of the density operator by orthogonal tensor operators $\mathcal{T}_{j}[16]$ (spherical or cartesian tensors) one can get an equation for expectation values $\left\langle\mathcal{T}_{j}\right\rangle$ in the weak colision approximation:

$$
\begin{aligned}
& \frac{\mathrm{d}\left\langle\mathcal{T}_{j}\right\rangle}{\mathrm{d} t}=-\sum_{k} A_{j k}\left\langle\mathcal{T}_{k}\right\rangle-\sum_{k} R_{j k}\left(\left\langle\mathcal{T}_{k}\right\rangle-\left\langle\mathcal{T}_{k}\right\rangle_{\text {eq }}\right) \\
& R_{j k}=\frac{1}{2} \int_{-\infty}^{+\infty} \operatorname{Tr}\left\{\left[\mathcal{T}_{j}, \tilde{\mathcal{H}}(t)\right]\left[\mathcal{T}_{k}, \tilde{\mathcal{H}}(t+\tau)\right]^{+}\right\} \mathrm{d} \tau,
\end{aligned}
$$

where $A_{j k}$ and $R_{j k}$ are spin dynamics [16] and relaxation matrixes respectively and $\tilde{\mathcal{H}}(t)$ is the perturbation Hamiltonian in RTF.

The diagonal elements, $R_{j j}=R_{j}$, of the relaxation matrix are the relaxation rates for the observables $\left\langle\mathcal{T}_{j}\right\rangle$ whereas $R_{j k}$ for $j \neq k$ are the cross relaxation rates between $\left\langle\mathcal{T}_{j}\right\rangle$ and $\left\langle\mathcal{T}_{k}\right\rangle$. Taking $\mathcal{T}_{j}=\frac{1}{c}\left(\mathcal{I}_{x}, \mathcal{I}_{z}\right)$, where $c^{2}=\operatorname{Tr}\left(\mathcal{I}_{x}\right)^{2}=\operatorname{Tr}\left(\mathcal{I}_{z}\right)^{2}$, the effective relaxation times $T_{2 e}$ and $\tilde{T}_{1 e}$ can be calculated, where

$$
\frac{1}{T_{2 \mathrm{e}}}=\frac{1}{2 \operatorname{Tr}\left(\mathcal{I}_{x}^{2}\right)} \int_{-\infty}^{+\infty} \operatorname{Tr}\left\{\left[\mathcal{I}_{x}, \ddot{\mathcal{H}}(\dot{v})\right]\left[\mathcal{I}_{x}, \tilde{\mathcal{H}}(t+\tau)\right]^{+}\right\} \mathrm{d} \tau .
$$

From Eq. 4 one can easily obtain the general expression for $1 / T_{1}$ by inserting operator $\mathcal{I}_{z}$ instead of $\mathcal{I}_{x}$.

In this paper we shall calculate $T_{2 \mathrm{e}}$ due to the fluctuating part of spin interactions. One should note that the static part of spin interactions can also contribute to the relaxation rate. This effect will be discussed elsewhere.

Consider a system of identical nuclear spins $\mathcal{I}_{i}=\mathcal{I}$ in a strong magnetic field $B_{0}$ along $z$ axis, subject to a periodic train of very short r.f pulses (delta pulses):

$$
90_{90^{\circ}}^{\circ}-\left[-\tau_{0}-\left(\theta_{1}\right)_{\phi_{1}}-\tau_{1}-\left(\theta_{2}\right)_{\phi_{2}}-\tau_{2}-\cdots-\left(\theta_{N}\right)_{\phi_{N}}-\tau_{N}\right]_{n}
$$

at resonance îrequency $\omega_{0}=\gamma B_{0}$, where $\theta_{k}$ and $\phi_{k}$ are nutation and phase angles of the pulses with repetition period of the cycle $T_{c}=\sum_{k=0}^{N} \tau_{k}$.

After following transformations into rotating frame (RF) and RTF [6] the time dependent spin Hamiltonian $\tilde{\mathcal{H}}(t)$ for dipolar and quadrupole interaction of axial symmetry may be presented in the form:

$$
\begin{gathered}
\tilde{\mathcal{H}}(t)=\sum_{i j} \sum_{m} \tilde{T}_{2 m}\left(\mathcal{I}_{i} \mathcal{I}_{j}\right) X_{m}^{i j *}(t), \\
X_{r i}^{i j}=b_{i j}\left(C_{2 m}\left(\theta_{i j}(t), \phi_{i j}(t)\right)-\left\langle C_{2 m}\left(\theta_{i j}(t), \phi_{i j}(t)\right)\right\rangle\right) \\
\tilde{T}_{2 m}\left(\mathcal{I}_{i} \mathcal{I}_{j}\right)=\sum_{m^{\prime}} \mathcal{D}_{m m^{\prime}}(\Omega(t)) e^{\mathrm{i} m \omega_{I} t} T_{2 m^{\prime}}\left(\mathcal{I}_{i} \mathcal{I}_{j}\right) \\
T_{2 m}\left(\mathcal{I}_{i} \mathcal{I}_{j}\right)=\left(\mathcal{I}_{i} \nabla\right)\left(\mathcal{I}_{j} \nabla\right)\left(r^{2} C_{2 m}(\theta, \phi)\right)
\end{gathered}
$$




$$
\begin{gathered}
C_{2 m}(\theta, \phi)=\sqrt{\frac{4 \pi}{5}} Y_{2 m}(\theta, \phi), \\
b_{i j}=\frac{\mu_{0}}{4 \pi} \gamma^{2} \hbar r_{i j}^{-3}, \quad b_{i i}=\frac{e Q q_{i}}{2 I(2 I-1) \hbar},
\end{gathered}
$$

where $T_{2 m}\left(\mathcal{I}_{i}, \mathcal{I}_{j}\right), Y_{2 m}(\theta, \phi)$ and $C_{2 m}(\theta, \phi)$ are spherical tensors (unnormalized), spherical functions and Racah functions of second rank.

$\mathcal{D}_{m m^{\prime}}(\Omega(t)) \equiv \mathcal{D}_{m m^{\prime}}^{(2)}(\Omega(t))$ is Wigner rotation matrix for second rank tensor, $\Omega(t) \equiv(\alpha(t), \beta(t), \gamma(t))$ are Euler angles of resultant rotations of RTF during multipulse sequence and $b_{i j}, b_{i i}$ are dipolar and quadrupole coupling constants respectively.

We assume that orientation of RTF and spins relative to RF is fixed between following pulses, i.e. $\mathcal{D}_{m m^{\prime}}(\Omega(t))$ is picewise constant function of time:

$$
\mathcal{D}_{m m^{\prime}}(\Omega(t))=\sum_{k=0}^{N-1} P_{k}(t) \mathcal{D}_{m m^{\prime}}\left(\Omega_{k}\right),
$$

where $P_{k}(t)$ are square pulses of the width $\tau_{k}$, which can be expanded in Fourier series:

$$
P_{k}(t)=\sum_{n=-\infty}^{\infty} c_{k n} e^{\mathrm{i} n \omega_{c} t}
$$

where $\omega_{c}=2 \pi / T_{c}$ is cycle frequency. From Eq. 11 and Eq. 12 one gets:

$$
\mathcal{D}_{m m^{\prime}}(\Omega(t))=\sum_{n} C_{m m^{\prime}}^{n} e^{i n \omega_{c} t},
$$

where

$$
C_{m m^{\prime}}^{n}=\sum_{k} c_{k n} \mathcal{D}_{m m^{\prime}}\left(\Omega_{k}\right)
$$

Using commutation relations for spherical tensors [17, 18], orthogonality of spherical tensors and functions and assuming exponential correlation function with correlation time $\tau_{c}$ one gets from Eqs. 4-14 the following expressions which are valid for sufficiently small values of a verage r.f. field, i.e. when $\gamma_{I}^{2} \overline{B_{1}^{2}} \tau_{c}^{2} \ll 1$ :

$$
\begin{aligned}
\frac{1}{T_{1 \mathrm{e}}}= & \frac{\Delta M_{2}}{6} \sum_{m m^{\prime} n} m^{\prime 2}\left|C_{m m^{\prime}}^{n}\right|^{2} \kappa_{m} J_{m}\left(m \omega_{I}+n \omega_{c}\right), \\
\frac{1}{T_{2 \mathrm{e}}}= & \frac{\Delta M_{2}}{12} \sum_{m m^{\prime} n} \kappa_{m} J_{m}\left(m \omega_{I}+n \omega_{c}\right)\left(\left|C_{m m^{\prime}}^{n}\right|^{2} \lambda_{m^{\prime}}\right. \\
& \left.+C_{m m^{\prime}}^{n} C_{m m^{\prime}+2}^{n *} \lambda_{m^{\prime}}^{+}+C_{m m^{\prime}}^{n} C_{m m^{\prime}-2}^{n *} \lambda_{m^{\prime}}^{-}\right), \\
J_{m}\left(\omega, \tau_{c}\right)= & \frac{\int_{-\infty}^{\infty}\left\langle X_{m}^{i j}(t) X_{m}^{i j *}(t+\tau)\right\rangle e^{\mathrm{i} \omega \tau} d \tau}{\left\langle\left|X_{m}^{i j}\right|^{2}\right\rangle}=\frac{2 \tau_{c}}{1+\omega^{2} \tau_{c}^{2}},
\end{aligned}
$$




$$
\begin{gathered}
\Delta M_{2}=\left(M_{2}\right)_{R L}-\left(M_{2}\right)_{\text {motion }}=\frac{9}{4} N_{I}^{-1} \sum_{i, j=1}^{N_{I}}\left\langle\left|X_{0}^{i j}\right|^{2}\right\rangle \\
\kappa_{m}=\frac{\left\langle\left|X_{m}^{i j}\right|^{2}\right\rangle}{\left\langle\left|X_{0}^{i j}\right|^{2}\right\rangle} \\
\lambda_{m^{\prime}}=6-m^{\prime 2} \\
\lambda_{m^{\prime}}^{ \pm}=\frac{1}{2} \sqrt{\left(1 \mp m^{\prime}\right)\left(2 \mp m^{\prime}\right)\left(3 \pm m^{\prime}\right)\left(4 \pm m^{\prime}\right)}
\end{gathered}
$$

where $J_{m}^{i j}(\omega)=J_{m}(\omega)$ is the reduced spectral density of the correlation function which is assumed to be independent of $i, j$ and $\Delta M_{2}$ is change of the second moment of the resonance line in the case of motional narrowing (compared with $M_{2}$ for rigid lattice). In further considerations we shall limit our discussion only to $T_{2 \mathrm{e}}$. For simplicity we can drop the factor $\kappa_{m}$, which is unity in isotropic systems, or we can introduce it into definition of $J_{m}(\omega)$. Using orthogonality of the spherical functions and square pulse functions $P_{k}(t)$ and assuming $\omega_{I} \gg \omega_{c}$ one can present expresion for $T_{2 \mathrm{e}}$ in a useful form:

$$
\begin{gathered}
\frac{1}{T_{2 \mathrm{e}}}=\frac{\Delta M_{2}}{12} \sum_{m m^{\prime}} \sum_{k=0}^{N-1} \frac{\tau_{k}}{T_{c}} d_{m m^{\prime}}\left(\beta_{k}\right)\left[d_{m m^{\prime}}\left(\beta_{k}\right) \lambda_{m^{\prime}}+\cos 2 \gamma_{k} d_{m m^{\prime}+2}\left(\beta_{k}\right) \lambda_{m}^{\prime}+\right] \\
\times J_{m}\left(m \omega_{I}\right)-\Gamma \\
\Gamma=\frac{\Delta M_{2}}{12} \sum_{m^{\prime} k k^{\prime} n=-\infty} \sum_{n=1}^{+\infty} \frac{c_{k n} c_{k^{\prime} n} n^{2} \tau_{c}}{n^{2}+\left(\omega_{c} \tau_{c}\right)^{-2}} \mathcal{D}_{0 m^{\prime}}\left(\Omega_{k}\right) \\
\times\left[\mathcal{D}_{0 m}^{*}\left(\Omega_{k^{\prime}}\right) \lambda_{m^{\prime}}+\mathcal{D}_{0 m^{\prime}+2}^{*}\left(\Omega_{k^{\prime}}\right) \lambda_{m^{\prime}}^{+}+\mathcal{D}_{0 m^{\prime}-2}^{*}\left(\Omega_{k^{\prime}}\right) \lambda_{m^{\prime}}^{-}\right]
\end{gathered}
$$

where $d_{m m^{\prime}}(\beta)=\mathcal{D}_{m m^{\prime}}(0, \beta, 0)$ and $\Omega_{k}=\left(\alpha_{k}, \beta_{k}, \gamma_{k}\right)$.

In the case of r.f. pulses with fixed phase $\phi_{k}=\phi$ one gets $\Omega_{k}=(\phi-$ $\left.\pi / 2, \beta_{k}, \pi / 2-\phi\right)$.

Using Eqs. (22) and (23) we shall consider several sequences which are commonly used in NMR. In the case of Ostroff-Waugh-Mansield-Ware sequences extended for arbitrary angles, $\theta=2 \pi P / N$, by Rhim [13] and Vega and Vaughan [14], $(\pi / 2)_{y}-\left[-\tau-\theta_{x}-2 \tau-\theta_{x}-\cdots-2 \tau-\theta_{x}-\tau\right]_{n}, \tau_{k}=2 \tau, T_{c}=2 N \tau$ with $y, x$ corresponding to the angles $\phi_{0}=\pi / 2, \phi_{k}=0$ and $\Omega_{0}=(0,0,0), \Omega_{k}=(-\pi / 2, k \theta, \pi / 2)$ for $k \neq 0$ one gets:

$$
\begin{array}{r}
\frac{1}{T_{2 \mathrm{e}}}=\frac{1}{T_{2}}-\Gamma=\frac{\Delta M_{2}}{3}\left(3 \tau_{c}-\frac{5 \tau_{c}}{1+\omega_{0}^{2} \tau_{c}^{2}}+\frac{2 \tau_{c}}{1+4 \omega_{0}^{2} \tau_{c}^{2}}\right) \\
-\Delta M_{2} \tau_{c} \frac{\tanh u}{u} \frac{\sin ^{2} \theta}{1-\left(\frac{\cos \theta}{\cosh u}\right)^{2}},
\end{array}
$$

where $T_{2}$ is spin-spin relaxation time and $u=\tau / \tau_{c}$. 
For a second extended sequence OWMW, $(\pi / 2)_{y}-\left[-\tau-\theta_{x}-2 \tau-\theta_{-x}-\tau\right]_{n}$, with arbitrary $\theta, \Omega_{0}=(0,0,0)$ and $\Omega_{1}=(-\pi / 2, \theta, \pi / 2), \tau_{k}=2 \tau$ and $T_{c}=4 \tau$ one gets:

$$
\frac{1}{T_{2 \mathrm{e}}}=\frac{1}{T_{2}}-\Delta M_{2} \tau_{c} \frac{\tanh u}{u} \sin ^{2} \theta .
$$

Taking subtraction of Eqs. (25) and (26) one gets the diference of the relaxation rates for these two sequences:

$$
\delta=\Delta M_{2} \tau_{c} \frac{\tanh u}{u} \frac{\sin ^{2} \theta \cos ^{2} \theta}{\cosh ^{2} u-\cos ^{2} \theta} .
$$

This difference has maximum value for $\cos \theta_{\max }=(1+\tanh u)^{-1 / 2}$. In the case of fast motion, when $u=\tau / \tau_{c} \gg 1$ one gets maximum value of $\delta$ for $\theta_{\max }=\frac{\pi}{4}$ and $\frac{3 \pi}{4}$ whereas for slow motion, when $u \ll 1$, one gets maximum $\delta$ for $\theta_{\max }=u^{1 / 2}$ and $\pi-u^{1 / 2}$. In the case of $\theta=\pi / 2$ one gets $\delta=0$, i.e. relaxation time $T_{2 \mathrm{e}}$ for both sequences is the same as predicted earlier [7] for $m=0$ whereas for CPMG sequence [19-20] one gets $T_{2 \mathrm{e}}=T_{2}$.

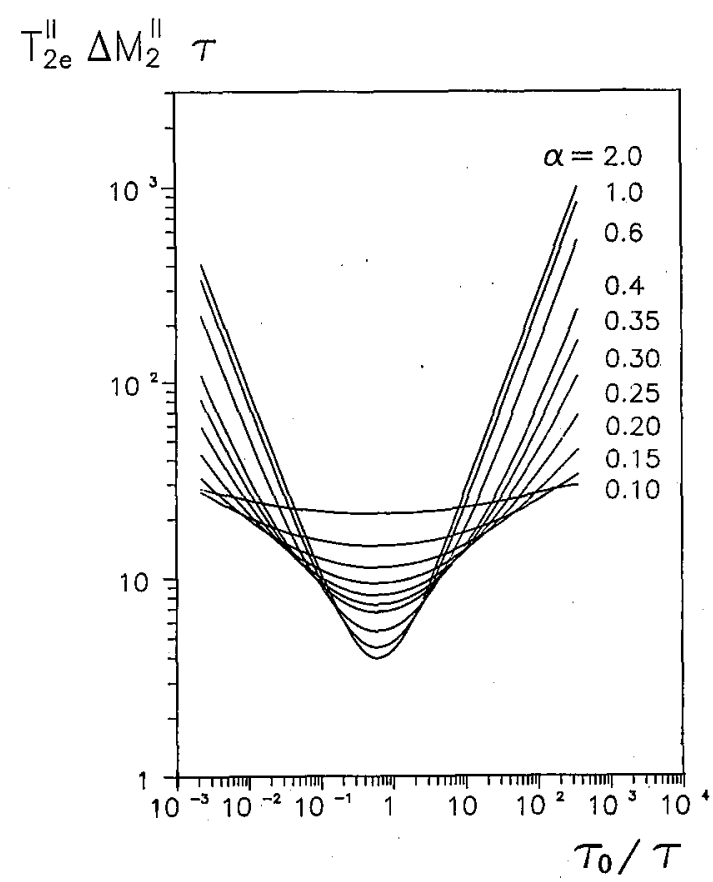

Fig. 1. Effective spin-spin relaxation time $T_{2 \mathrm{e}}$ in the presence of Log-Gauss distribution of correlation times and fast spin exchange as a function of $\tau_{0} / \tau$. Resonance frequency was $60.5 \mathrm{MHz}$. 


\section{Spin-spin relaxation in the presence of continuous distribution of the correlation times}

Consider a system consisted of some spin subsystems, each of them characterized by relative spin population $p_{k}$ and effective relaxation time $\left(T_{2 \mathrm{e}}\right)_{k}$. According to the Zimmerman theory [21], in the case of fast exchange or fast spin diffusion one can write:

$$
\frac{1}{T_{2 \mathrm{e}}}=\sum_{k} p_{k} \frac{1}{\left(T_{2 \mathrm{e}}\right)_{k}}, \quad \sum_{k} p_{k}=1 .
$$

In the presence of a continuous distribution of correlation times, described by a function $g\left(\tau_{c}\right)$, Eq. (26) may be written in the form:

$$
\frac{1}{T_{2 \mathrm{e}}}=\int_{0}^{\infty} \frac{g\left(\tau_{c}\right)}{T_{2 \mathrm{e}}\left(\tau_{c}\right)} \mathrm{d} \tau_{c}, \quad \int_{0}^{\infty} g\left(\tau_{c}\right) \mathrm{d} \tau_{c}=1 .
$$

It has been shown in earlier papers $[22,23]$ that results of spin-lattice relaxation time measurements in some biological systems can be interpreted assuming, so called, Log-Gauss (logarithmic-Gaussian) distribution of the correlation times $f(s)[24]$ :

$$
f(s)=g\left(\tau_{c}\right) \frac{d \tau_{c}}{d s}=\frac{\alpha}{\sqrt{\pi}} \mathrm{e}^{\alpha^{2} s^{2}}, \quad s=\ln \frac{\tau_{c}}{\tau_{0}},
$$

where $\tau_{0}$ is the mean correlation time and $\alpha$ is a parameter determining the width of the distribution.

From Eqs. (27) and (28) one can get an expression for average spin-spin effective relaxation time $T_{2 e}$, in the presence of $\log -$ Gauss distribution of correlation times and fast spin exchange:

$$
\frac{1}{T_{2 \mathrm{e}}}=\int_{-\infty}^{\infty} \frac{f(s)}{T_{2 \mathrm{e}}(s)} \mathrm{d} s
$$

Effective spin-spin relaxation times $T_{2 e}$, as a function of $\tau_{0} / \tau$, have been numerically calculated from (29) for different values of parameter $\alpha$. Results of these calculations are presented in Fig. 1.

Assuming Arrhenius temperature dependence of $\tau_{0}$ one can see from this figure that temperature dependence of $T_{2 \mathrm{e}}$ flattens with increasing width of the distribution of correllation times.

In the case of slow exchange between spin subsystems one can write the relaxation function in the form:

$$
R(t)=\sum_{k} p_{k} e^{-t /\left(T_{2 e}\right)_{k}}
$$

In the presence of continuous Log-Gauss distribution of correlation times one can write:

$$
R(t)=\int_{-\infty}^{\infty} f(s) \mathrm{e}^{-t / T_{2 e}(s)} \mathrm{d} s
$$



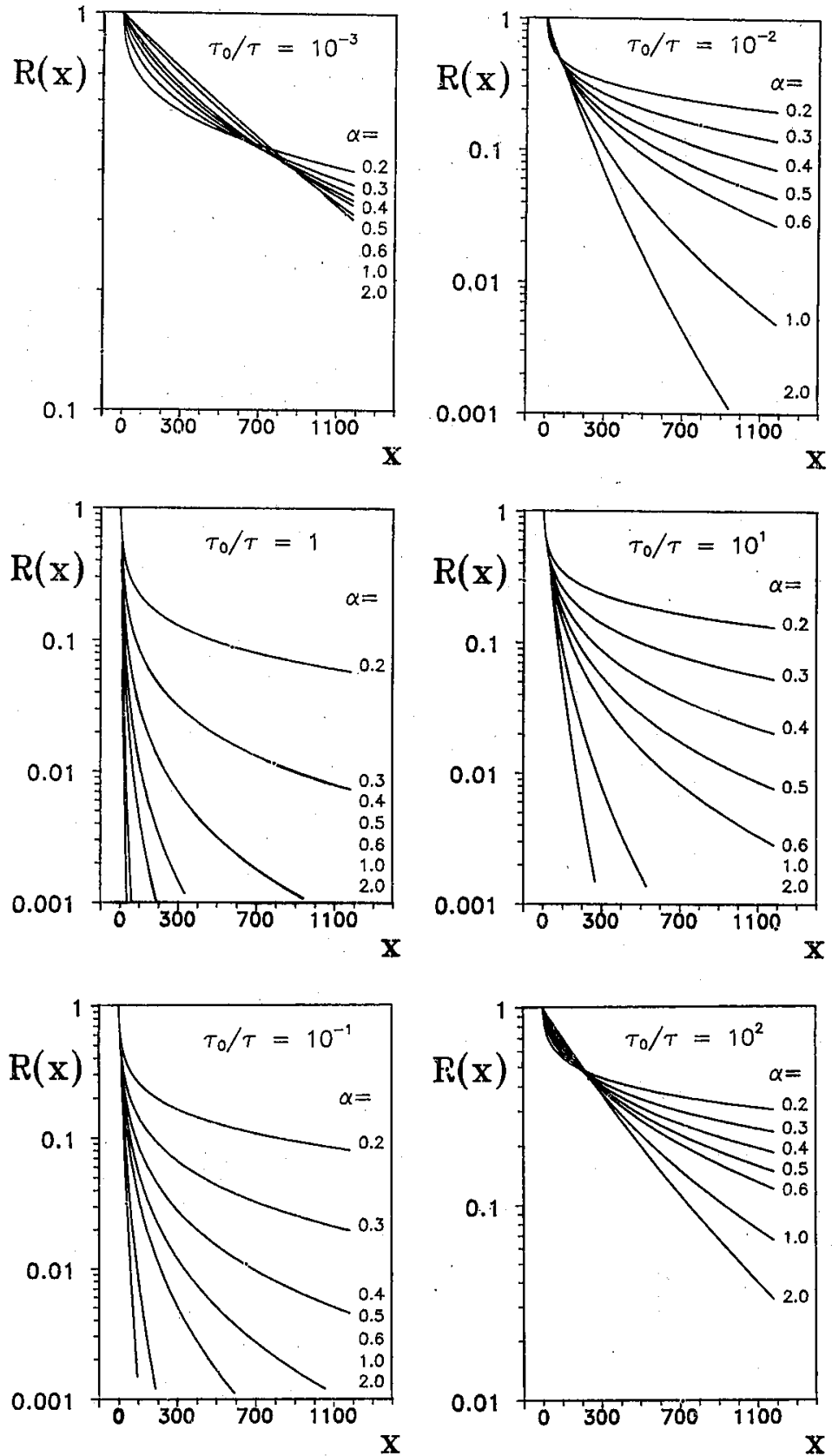

Fig. 2. Relaxation function in the presence of Log-Gauss distribution of correlation times and slow spin exchange as a function of $x=\Lambda M_{2} r t$. Calculations have been done for OWMW pulse sequence with $\theta=\pi / 2$ (Eq. 24). Resonance frequency was 60.5 $\mathrm{MHz}, \tau=10^{-5} s, \Delta M_{2}=10^{9} \mathrm{~s}^{-2}$. 
The results of the numerical calculations of the relaxation function $R(t)$ in the case of slow spin exchange for different values of $\tau_{0} / \tau$ and $\alpha$ are presented in Fig. 2 . As can be expected, for small width of the distribution the relaxation function is well described by one exponential decay. With increasing distribution width nonexponentiality of the relaxation function becomes more and more evident.

\section{Acknowledgements}

The author (JSB) wishes to thank Professor M. Bloom for helpful discussion.

\section{References}

[1] E.D. Ostroff, J.S. Waugh, Phys. Rev. Lett. 16, 1097 (1966).

[2] P. Mansfield, D. Ware, Phys. Lett. 22, 133 (1966).

[3] J.S. Waugh, C.H. Wang, Phys. Rev. 162, 209 (1967).

[4] P. Mansfield, D. Ware, Phys. Rev. 168, 318 (1968).

[5] J.S. Waugh, L.M. Huber, U. Haeberlen, Phys. Rev. Lett. 20, 180 (1968).

[6] U. Haeberlen, J.S. Waugh, Phys. Rev. 185, 420 (1969).

[7] W. Gründe, H. Schmiedel, D. Freude, Ann. Phys. (Leipzig) 27, 409 (1971).

[8] P. Mansfield, Prog. Nucl. Magn. Resonance Spectrosc. 8, 43 (1971).

[9] A.N. Garroway, P. Mansfield, A.D. Stalker, Phys. Rev. B 11, 121 (1975).

[10] R. Müller, R. Wilsh, J. Magn. Resonance 21, 135 (1976).

[11] A. Jasiński, M. Stachurowa, Acta Phys. Pol. A55, 433 (1979).

[12] J.S. Blicharski, Can. J. Phys. 64, 733 (1986).

[13] W. -K. Rhim, .P. Burum, A.D. Eleman, J. Chem. Phys. 68, 692 (1978).

[14] A.J. Vega, R.W. Vaughan, J. Chem. Phys. 68, 1958 (1978).

[15] A. Abragam, The Principles of Nuclear Magnetism, Claredon Press, Oxford, 1961.

[16] M. Bloom, in: Proceedings of the International School of Physics. Physics of NMR Spectroscopy in Biology and Medicine ed. B. Maraviglia, North-Holland, Amsterdam-Oxford-New York-Tokyo 1988.

[17] A.R. Edmonds, Angular Momentum in Quanlum Mechanics, Princeton University Press, Princeton N.Y. 1957.

[18] E.P. Wigner, Group Theory, Academic Press, New York 1959.

[19] H.Y. Carr, E.M. Purcell, Phys. Rev. 94, 630 (1954).

[20] S. Meiboom, D. Gill, Rev. Sci. Instr. 29, 688 (1958).

[21] J.R. Zimmerman, W.E. Brittin, J. Phys. Chem. 61, 1328 (1957).

[22] L.J. Lynch, K.H. Marsden, E.P. George, J. Chem. Phys. 51, 5673 (1969).

[23] B. Blicharska, Z. Florkowski, J.W. Hennel, G. Held, F. Noack, Biochim. Biophys. Acta 207, 381 (1970).

[24] B. Blicharska, J.S. Blicharski, Acta Phys. Pol. A41, 347 (1972). 